\title{
CB2R orchestrates neuronal autophagy through regulation of the mTOR signaling pathway in the hippocampus of developing rats with status epilepticus
}

\author{
QIONG WU ${ }^{1}$, MIAO ZHANG ${ }^{2}$, XUEYAN LIU $^{1}$, JUNMEI ZHANG ${ }^{1}$ and HUA WANG ${ }^{1}$ \\ ${ }^{1}$ Department of Pediatric Neurology, Shengjing Hospital of China Medical University, Shenyang, Liaoning 110004; \\ ${ }^{2}$ Department of Forensic Pathology, China Medical University School of Forensic Medicine, \\ Shenyang, Liaoning 110122, P.R. China
}

Received February 15, 2019; Accepted November 29, 2019

DOI: $10.3892 /$ ijmm.2019.4439

\begin{abstract}
Neuronal loss and gliosis are the major pathological changes after status epilepticus (SE). The authors' previous study revealed the time-dependent changes of cannabinoid receptor type 2 (CB2R) in hippocampal neurons of developing rats after SE, which were accompanied by a decrease in the number of neurons. Meanwhile, growing evidence indicates that $\mathrm{CB} 2 \mathrm{R}$ stimulation exerts anti-convulsant properties in seizure models. However, the activation of CB2R in neuronal repair in response to the damage after SE is still unclear. In this experiment, a highly-selective CB2R agonist JWH133 and antagonist AM630 were administered to determine the activity of CB2R in neuronal autophagy and apoptosis of the post-SE repair in developing rats. The present results revealed that activation of CB2R by JWH133, not only obviously lowered the success rate, 24-h death rate and the Racine stage in the model, but also extended the latency period to SE. In addition, compared with the vehicle control group, CB2R activation increased neuronal autophagy and the expression of phosphorylated-mammalian target of rapamycin (p-mTOR)/mTOR, Beclin-1, and LC3II/LC3I while decreasing the expression of p-Unc-51-like autophagy-activating kinase 1 (ULK-1)/ULK1, p62, and cleaved caspase-3. These results were dose-dependent and were especially evident in the high-dose group, and interestingly the opposite results were obtained in the AM630 group. Thus, CB2R orchestrates neuronal autophagy through regulation of the mTOR signaling pathway in the hippocampus of developing rats with SE. These findings might provide an important basis for further investigation of the therapeutic role of CB2R in ameliorating epilepsy-related neuronal damage.
\end{abstract}

Correspondence to: Professor Hua Wang, Department of Pediatric Neurology, Shengjing Hospital of China Medical University, 36 Sanhao Street, Heping, Shenyang, Liaoning 110004, P.R. China E-mail: wanghcmu@163.com

Key words: cannabinoid receptor type 2, hippocampus, autophagy, apoptosis, mammalian target of rapamycin, status epilepticus

\section{Introduction}

Epilepsy is the term used to describe a common neurological disorder characterized by long-term, recurrent epileptic seizures that is accompanied by various neurobiological, cognitive, psychological and social consequences (1). Temporal lobe epilepsy (TLE) is one of the most common forms of partial epilepsy and its animal model can be divided into acute, silent, and chronic phases (2). Abundant alterations can be observed in the hippocampus during the acute and silent period after status epilepticus (SE), such as neuronal loss and degeneration, gliosis, mossy fiber sprouting, scattering of dentate granule cells, and synaptic modifications in the dentate gyrus, all of which contribute to the epileptogenic process $(3,4)$. The accumulated evidence shows that neuronal cell death in the hippocampus is the most important factor in initiating and precipitating the development of epileptic disorders (5-7). In addition, previously discovered datum also provided novel insight into two mechanisms in connection with cell survival and death, autophagy and apoptosis, and their function in regulating neuronal fate (8-10).

Autophagy plays a vital role in various aspects of cell homeostasis, such as cell survival, cell death, cell metabolism, development, neuroprotection and even neurodegeneration (11). Mammalian target of rapamycin (mTOR) is a central regulator of autophagy that strategically influences synaptic plasticity at pre- and postsynaptic sites by regulating the balance between protein synthesis and degradation (12-15). Activation of mTOR can inactivate assembly of Unc-51-like autophagy-activating kinase 1 (ULK-1) at S757 and in turn prevent phosphorylation and activation of Beclin-1 at S14 to halt autophagy $(16,17)$. Beclin-1 promotes autophagosome nucleation, through the generation of PI3P in complex with a class III phosphatidylinositol 3 kinase. Autophagosome maturation and the lipidation of LC3-II to the autophagosome membrane is completed by two ubiquitin-like conjugation systems [ATG5-ATG12-ATG16L and (ATG8) LC3] $(18,19)$. The limiting autophagosome membrane elongates to recognize and encapsulate degradation substrates via p62 protein (20). Several findings have implied the participation of autophagy in progressive central nervous system (CNS) diseases such as Parkinson's, Huntington's 
and Alzheimer's disease $(21,22)$, amyotrophic lateral sclerosis $(23,24)$ and global ischemia (25). Furthermore, in the chronic phase of pilocarpine-induced seizures, cannabidiol post-treatment alleviates rat epileptic-related behaviors and activates the hippocampal cell autophagy pathway and antioxidant defense mechanisms (26). However, detailed understanding of the role played by autophagy in epilepsy is lacking.

The endogenous endocannabinoids and their receptors are important regulators of neuronal activity $(27,28)$. Cannabinoid receptor type 1 (CB1R), which is abundantly expressed throughout the CNS (29), has been a research focus for numerous years. In contrast, cannabinoid receptor type 2 (CB2R) is mainly distributed in immune organs and cells, including the spleen, lymphocytes and even neutrophil granulocytes $(30,31)$, and plays important roles in regulating immune function. Previous studies have paid much less attention to the role of CB2R in epilepsy, mainly because of its limited CNS expression. However, research has suggested that CB2R is expressed in the brain (32-34), raising the possibility that it could also play a direct role in mediating CNS function. Growing evidence shows that CB2R stimulation exerts anti-convulsant properties in seizure models. Previous studies suggested that activation of CB2Rs may regulate neuronal excitability in the hippocampus and increase excitatory synaptic transmission (35), trigger cell type-specific hippocampal pyramidal cell hyperpolarization (36), and suppress epileptic seizures (37). The author's previous study revealed the expression of CB2R in hippocampal neurons of developing rats and its time-dependent changes after SE, which are accompanied by a fall in the number of neurons (38). However, the activation of CB2R on neuronal repair in response to the damage after SE is still unclear. The present study hypothesized that the anti-convulsant effects of $\mathrm{CB} 2 \mathrm{R}$ during the neuronal damage-repair process may be contributing to induction of autophagy. In this experiment, a highly selective CB2R agonist JWH133 and antagonist AM630 was administered to verify the present hypothesis.

\section{Materials and methods}

Animal models. A total of 42 rats healthy male Sprague-Dawley rats (from the Medical Animal Center, Shengjing Hospital of China Medical University), 3 weeks of age and weighing 45.0-56.5 g, were used in this study. The rats were provided with ad libitum access to food and purified water and were maintained under controlled temperature, humidity, and lighting conditions $\left(20-25^{\circ} \mathrm{C}, 50-60 \%\right.$ humidity, 12 -h light/ dark cycle). The China Medical University Institutional Animal Care and Use Committee (no. 2016PS260K) approved all procedures.

A commonly used SE model was used, as previously reported by the authors' lab $(38,39)$. Male rats 3 -weeks old weighing 45.0-56.5 g were used. A total of 42 rats were randomly divided into 7 groups ( $\mathrm{n}=6$ per group): Control group and $2 \mathrm{~h}, 1,3,7,14$ and $21 \mathrm{~d}$ groups. Furthermore, 30 male rats were randomly divided into 5 groups ( $n=6$ per group) according to pre-treatment: SE group, Vehicle group, JWH133 $1 \mu \mathrm{g} /$ side group, JWH133 $3 \mu \mathrm{g} /$ side group and JWH133+AM630 group. Rats were treated with lithium chloride ( $3 \mathrm{mEq} / \mathrm{kg}$, intraperitoneal; Sigma-Aldrich; Merck KGaA) $16-18 \mathrm{~h}$ prior to the injection of pilocarpine and then methylscopolamine $(1 \mathrm{mg} / \mathrm{kg}$, intraperitoneal; Sigma-Aldrich; Merck KGaA) $30 \mathrm{~min}$ prior to injection of pilocarpine for reducing the peripheral cholinergic effects. Experimental animals were then injected intraperitoneally (i.p.) with a single dose of $30 \mathrm{mg} / \mathrm{kg}$ of pilocarpine in order to induce SE. Control rats were administered a comparable volume of vehicle after the initial methylscopolamine treatment. Behavioral observation after pilocarpine injection was performed and pilocarpine-induced seizures were evaluated according to the Racine scale (40). Seizure intensity was that stage 1 (facial clonus with scratching, chewing and grooming), stage 2 (head nodding, tail wagging and chirping), stage 3 (forelimb clonus, wet dog shakes, standing tonus, and occasional loss of posture), stage 4 (forelimb clonus with rearing without falling), and stage 5 (four limb clonus with rearing and falling or jumping). SE was defined by continuous seizure activity for at least $30 \mathrm{~min}$ without full recovery between seizures when rats experienced continuous Stage 4 or 5 seizures. Then the rats were injected (i.p.) with $3 \%$ sodium pentobarbital $(30 \mathrm{mg} / \mathrm{kg})$ for anesthetization. After decapitation, each brain specimen was equally divided into two blocks. One block was used for morphological research and the other was used for molecular biological studies.

Pharmacological treatment. The CB2R agonist JWH133 ( $\mathrm{Ki}=3.4 \mathrm{nM}$ for CB2R; Tocris Bioscience) and the CB2R antagonist AM630 (Ki=31.2 $\mathrm{nM}$ for $\mathrm{CB} 2 \mathrm{R}$; Tocris Bioscience) were dissolved in $20 \%$ dimethyl sulfoxide (in normal saline). Subjects randomly received one intracerebroventricular injection dose of JWH133 (1 or $3 \mu \mathrm{g}$ in $1 \mu \mathrm{l} / \mathrm{side})$, AM630 (3 $\mu \mathrm{g}$ in $1 \mu \mathrm{l} / \mathrm{side})$, a mixed solution of $\mathrm{AM} 630(3 \mu \mathrm{g}$ in $1 \mu \mathrm{l} / \mathrm{side})$ and JWH133 (3 $\mu \mathrm{g}$ in $1 \mu \mathrm{l} / \mathrm{side})$, or vehicle ( $1 \mu \mathrm{l} / \mathrm{side}) 60 \mathrm{~min}$ before pilocarpine hydrochloride injection. In the present study, the SE group was used as the control group and injected with comparable volume of vehicle. All rats were administered lithium chloride-pilocarpine to induce SE. At 1 day after SE, six animals of each group were sacrificed.

Epileptic symptom observations after intracerebroventricular injection of CB2R agonist/antagonist in the lithium-pilocarpine SE model. After pilocarpine injection, the latency period to SE, Racine stage, success rate of the model and 24-h death rate after SE were examined to evaluate the potential of CB2R to remit seizure onset. The epileptic symptoms of each rat were observed and rated from 0 to 5 according to the Racine scale.

Immunofluorescence. For double-labelled immunofluorescence, $4-\mu \mathrm{m}$ brain sections were deparaffinized in xylene, hydrated with a graded alcohol series, blocked with 5\% non-immune fetal bovine serum (CAS: 9048-46-8; Sigma-Aldrich; Merck KGaA) in PBST (0.5\% Tween-20 in PBS) at $37^{\circ} \mathrm{C}$ for $2 \mathrm{~h}$ and incubated with primary antibodies overnight at $4^{\circ} \mathrm{C}$ with rabbit anti-CB2R antibody (1:100; cat. no. ab3561; Abcam), rabbit anti-mTOR antibody (1:200; cat. no. 20657-1-AP; Proteintech, Inc.), rabbit anti-LC3B antibody (1:400; cat. no. 2775; Cell Signaling Technology, Inc.), and 
mouse anti-NeuN antibody (1:800; cat. no. MAB377; EMD Millipore). Afterward, an Alexa Fluor 594-conjugated donkey anti-rabbit $\operatorname{IgG}$ (1:200; cat. no. ab150076; Abcam) and an Alexa Fluor 488-conjugated donkey anti-mouse IgG (1:200; cat. no. ab150105; Abcam) was used as secondary antibodies conjugated with CB2R and NeuN, mTOR and NeuN, or LC3B and NeuN. After washing with PBS three times, the sections were covered with the VECTASHIELD Mounting Medium glasses. For the evaluation of $\mathrm{CB} 2 \mathrm{R}^{+} / \mathrm{NeuN}^{+}$cells, $\mathrm{mTOR}^{+} /$ $\mathrm{NeuN}^{+}$cells and $\mathrm{LC}^{+} / \mathrm{NeuN}^{+}$cells, 10 microscope fields of the CA1, CA3, and DG regions were randomly selected under x200 magnification by fluorescence light microscopy (Nikon Eclipse 80i; Nikon Corporation).

TdT-mediated dUTP nick end labeling assay. As previously described (36), TdT-mediated dUTP nick end labeling (TUNEL) assay was performed for identifying neuronal death numbers. The assay was performed according to the manufacturer's protocol. TUNEL-positive cells were those with blue-green nuclear staining. For the evaluation of TUNEL-positive cells, 10 microscope fields of the CA1, CA3 and DG regions were randomly captured under x200 magnification by fluorescence light microscopy (Nikon Eclipse 80i) and the results were calculated as the means.

Protein extraction and western blotting assay. Protein preparation was performed as previously described (36). Hippocampal samples were homogenized with a sonicator in double-distilled water containing protease inhibitors and centrifuged at $14,000 \mathrm{x}$ g for $15 \mathrm{~min}$ at $4^{\circ} \mathrm{C}$, and the supernatant was collected. Protein concentrations were measured with the DC Protein Assay (Bio-Rad Laboratories, Inc.). Sample proteins $(40 \mu \mathrm{g})$ were denatured by heating at $90^{\circ} \mathrm{C}$ for $5 \mathrm{~min}$ and were size-fractionated by means of Bis-Tris $10 \%$ SDS-PAGE (Beyotime Institute of Biotechnology). Samples were then transferred to polyvinylidene fluoride (PVDF) membranes (EMD Millipore) and were blocked with 5\% fat-free milk for $2 \mathrm{~h}$ at room temperature. The membranes were incubated overnight at $4^{\circ} \mathrm{C}$ with the following primary antibodies: Rabbit anti-mTOR polyclonal antibody (1:1,000; cat. no. 20657-1-AP; Proteintech, Inc.), rabbit anti-p-mTOR monoclonal antibody (1:1,000; cat. no. 5536; Cell Signaling Technology, Inc.), rabbit anti-ULK1 polyclonal antibody (1:1,000; 20986-1-AP; Proteintech, Inc.), rabbit anti-p-ULK1 monoclonal antibody (1:1,000; cat. no. 14202; Cell Signaling Technology, Inc.), rabbit anti-Beclin-1 polyclonal antibody (1:1,000; cat. no. 11306-1-AP; Proteintech, Inc.), rabbit anti-p62 polyclonal antibody (1:1,000; cat. no. 18420-1-AP; Proteintech, Inc.), rabbit anti-LC3B monoclonal antibody (1:1,000; cat. no. 2775; Cell Signaling Technology, Inc.), and rabbit anti-GAPDH polyclonal antibody (1:5,000; cat. no. 10494-1-AP; Proteintech, Inc.). The following day, the membranes were washed and then incubated at room temperature for $2 \mathrm{~h}$ with the horseradish peroxidase conjugated goat anti-rabbit IgG antibody (1:5,000; cat. no. SA00001-2; Proteintech, Inc.). The membrane images were collected and analyzed with the Infrared Odyssey Imaging System. GAPDH was used as a control. Densitometric analysis of the bands was semiquantitatively performed using GEL-PRO 4.0 software (Media Cybernetics, Inc.).
Statistical analysis. All experiments were performed at least in triplicate. Data are reported as the mean \pm standard deviation and were analyzed using SPSS 18.0 for Windows (IBM, Corps). Multiple group comparisons were performed by one-way analysis of variance, followed by the LSD post hoc multiple comparison test. $\mathrm{P}<0.05$ was considered to indicate a statistically significant difference.

\section{Results}

Spatiotemporal expression changes in CB2R, $m T O R$ and LC3 in hippocampal neurons after SE. To determine the localization of neuronal CB2R, LC3B and mTOR and their expression changes in the hippocampus under normal conditions and after SE, double-label immunofluorescence and western blotting essays were performed. CB2R-, LC3B- and mTOR-positive neurons could be detected under normal conditions and after $\mathrm{SE}$ in the CA1, CA3, and DG regions of the hippocampus. CB2R and mTOR were mainly localized to the cytomembrane in neurons, with some occurring in the cytoplasm (Fig. 1A-C). In addition, the mTOR protein expression in all three regions was decreased at $2 \mathrm{~h}$ and 1 and 3 days after SE compared with the control group, whereas the expression levels of CB2R and LC3B were increased (Fig. 1D). Interestingly, the clearest differences in mTOR, CB2R and LC3B expression emerged 1 day after SE (Fig. 1D). Therefore, subsequent intervention experiments were all conducted 1 day after SE.

Effects of CB2R on epileptic symptoms after SE. The behavior of seizure activity was recorded following lithium-pilocarpine treatment. The rats began to exhibit definite SE behavior within 15-33 min after pilocarpine intraperitoneal injection. Significant differences could be observed in the success rate of the SE model between the vehicle group, JWH133 low-dose group and JWH133 high-dose group (Fig. 2A). Rats injected with low- or high-dose JWH133 showed a lower mortality rate compared with the vehicle group (Fig. 2B). The time between pilocarpine injection and seizures reaching Racine stage 4 or 5 was defined as the latency period. The latency periods of the JWH133 low-dose and high-dose groups were dose-dependently longer than that of the vehicle group (Fig. 2C). Rats injected with low- or high-dose JWH133 showed a significantly lower Racine stage compared with the vehicle group (Fig. 2D). Rats injected with low- or high-dose JWH133 showed alleviated epileptic symptoms compared with rats injected with vehicle or JWH-133+AM630. In addition, no significant difference could be observed in the success rate of the SE model, 24-h death rate, latency period and Racine stage between the SE and vehicle groups (Fig. 2). In each pilocarpine-treated group, rats with a Racine stage higher than 3 were included in the next two parts of the present study.

Hippocampal neuron apoptosis after SE. A TUNEL assay was performed to observe neuronal apoptosis in the hippocampus in each treatment group (Fig. 3A). The present study counted TUNEL-positive cell numbers in the CA1, CA3 and DG regions after SE. There were TUNEL-positive cells in all groups 1 day after SE and there was no significant difference in the average numbers of TUNEL-positive cells between the SE group and the vehicle group $(\mathrm{P}>0.05$; 
A
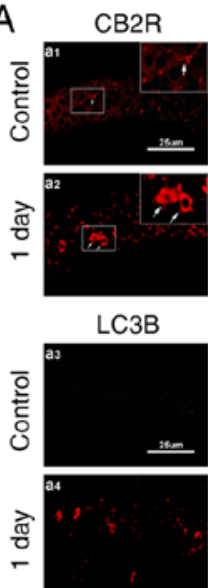

mTOR
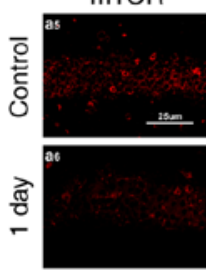

C
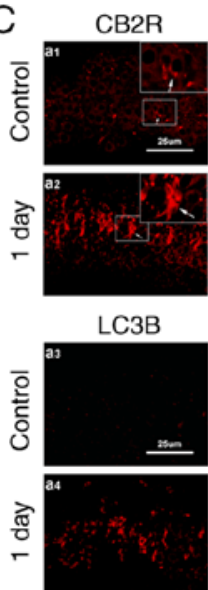

mTOR
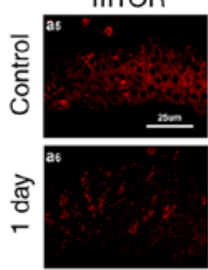

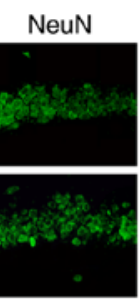

NeuN

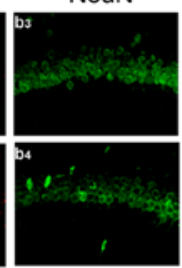

NeuN
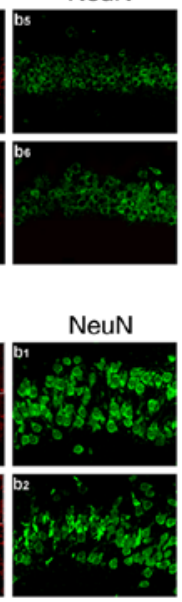

NeuN

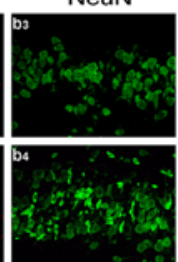

NeuN

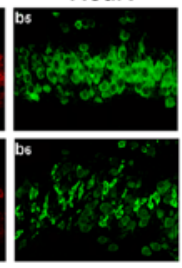

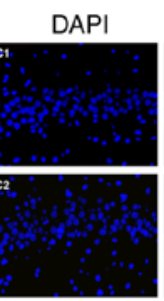

DAPI

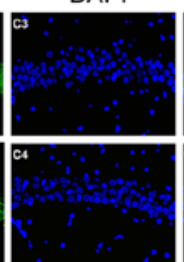

DAPI
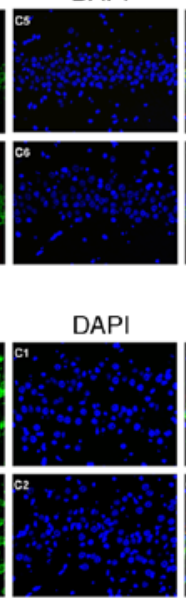

DAPI

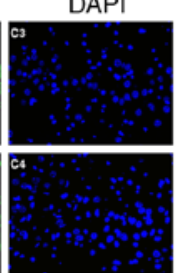

DAPI

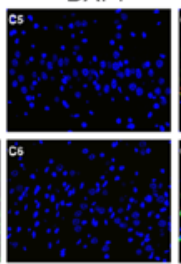

Merge

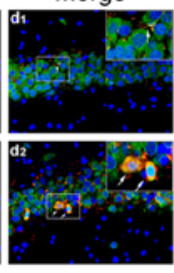

Merge

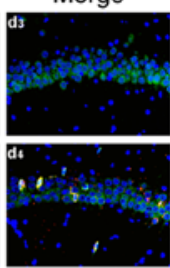

Merge

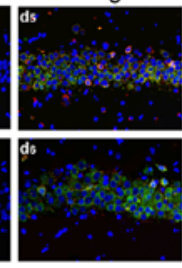

Merge

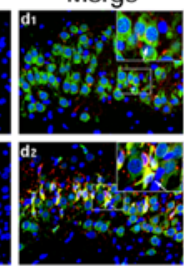

Merge

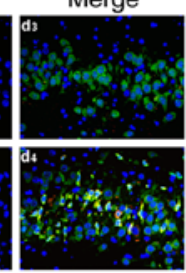

Merge
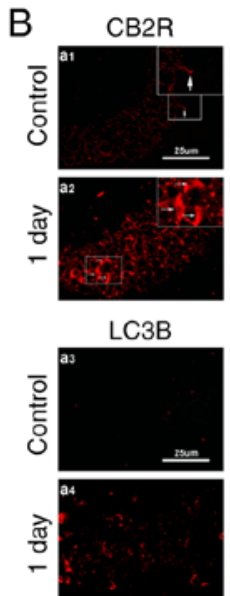

mTOR
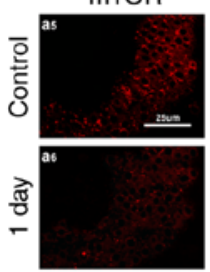

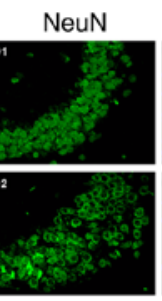

NeuN

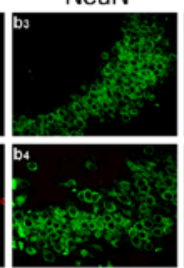

NeuN

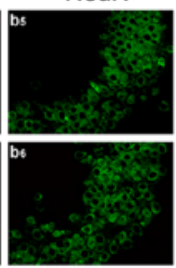

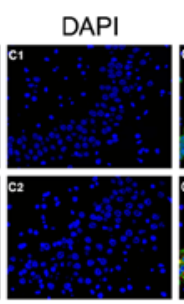

DAPI

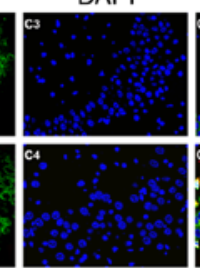

DAPI
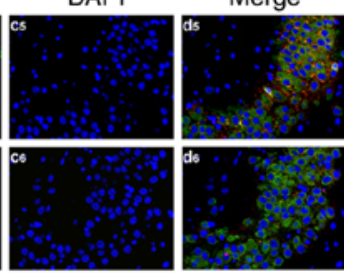

Merge

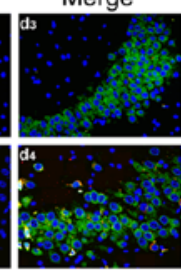

D
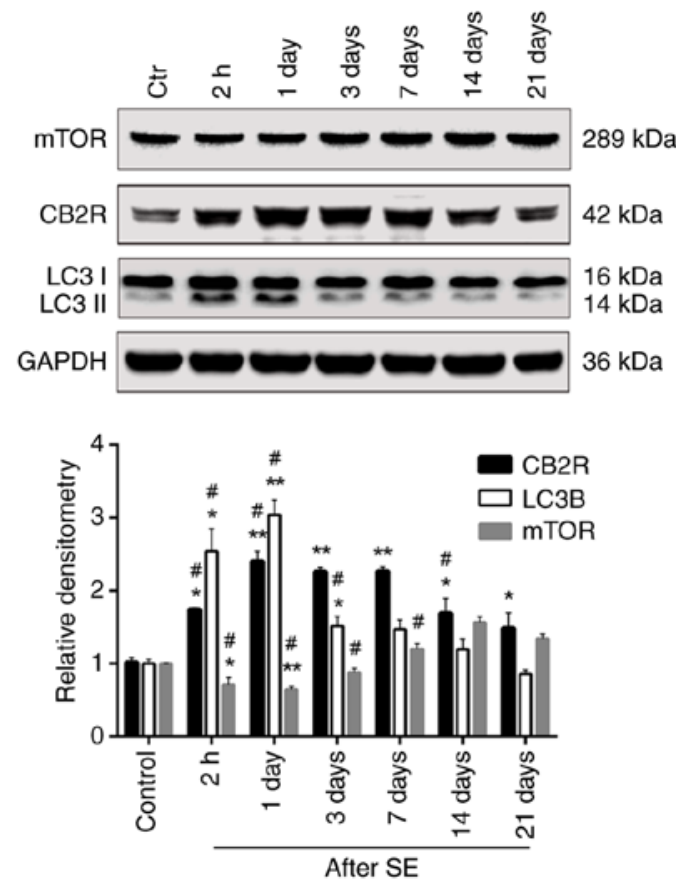

Figure 1. Spatiotemporal expression changes in CB2R, mTOR and LC3 in hippocampal neurons after status epilepticus. [(A) CA1, (B) CA3 and (C) DG regions)] Alexa Fluor 594-labeled $\mathrm{CB}_{2} \mathrm{R}^{+}, \mathrm{LC}_{3}{ }^{+}$and $\mathrm{mTOR}^{+}$cells in the hippocampal CA1, CA3, and DG regions (red). Alexa Fluor 488-labeled neuronal nuclear antigen NeuN (green). DAPI-labeled nucleus (blue). Images of NeuN marker with CB2R, LC3B and mTOR colocalization (yellow) were digitally merged within the CA1, CA3, and DG regions. (D) Western blot analysis of CB2R, LC3B, mTOR and GAPDH protein from hippocampal specimens. Data are presented as the mean \pm standard deviation. ${ }^{*} \mathrm{P}<0.05$ and ${ }^{* *} \mathrm{P}<0.01$ vs. the control group, $\mathrm{n}=6$ rats per group; ${ }^{*} \mathrm{P}<0.05$ vs. the previous group, $\mathrm{n}=6$ rats per group). mTOR, mammalian target of rapamycin; CB2R, cannabinoid receptor type 2.

Fig. 3B-D). However, the number of apoptotic neurons was seriously decreased by pretreatment with JWH133 at 1 day after SE. As shown in Fig. 3B-D, the numbers of apoptotic neurons were significantly and dose-dependently decreased in all three regions of the post-SE hippocampus in the JWH133-treated groups compared with the vehicle group 1 day after SE, whereas the numbers of apoptotic cells were increased again in the JWH133+AM630 group at 1 day after SE.

Effects of $C B 2 R$ on the expression of autophagy-related proteins in the hippocampus after SE. According to the authors' preliminary experiment (Fig. S1), JWH133 $1 \mu \mathrm{g}$ in $1 \mu \mathrm{l} /$ side and JWH133 $3 \mu \mathrm{g}$ in $1 \mu \mathrm{l} /$ side were selected as the 

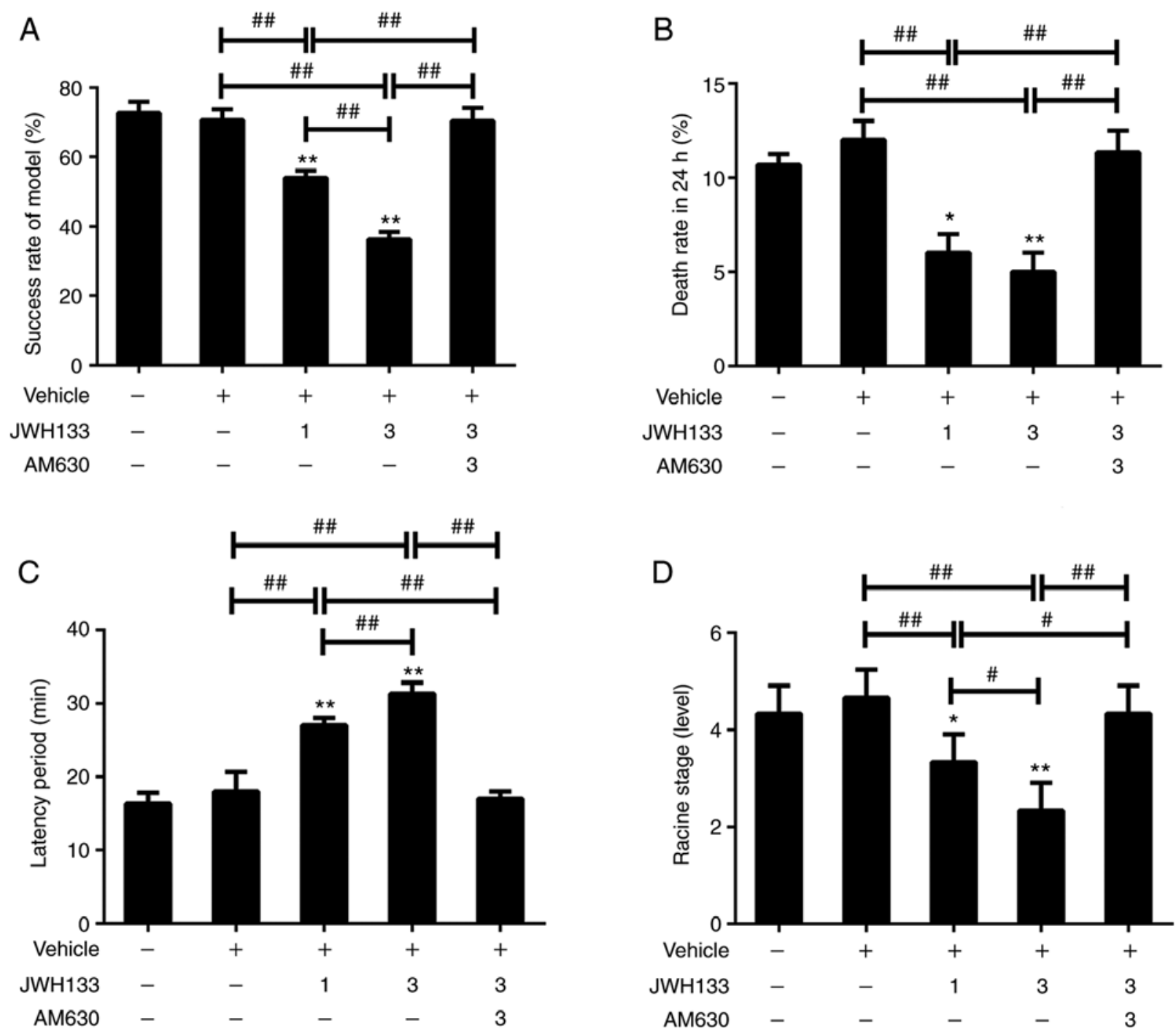

Figure 2. Protective effects of $\mathrm{CB} 2 \mathrm{R}$ in the status epilepticus model in vivo. (A) Activation of $\mathrm{CB} 2 \mathrm{R}$ dose-dependently decreased the success rate of the model. (B) Activation of CB2R decreased the 24-h death rate after pilocarpine injection. (C) Activation of CB2R dose-dependently increased the latency period. (D) Activation of CB2R dose-dependently decreased the Racine stage. The opposite effects were observed in the CB2R inhibition group administered AM630. Data are presented as the mean \pm standard deviation. ${ }^{*} \mathrm{P}<0.05$ and ${ }^{* * *} \mathrm{P}<0.01$ vs. the control group, ( $\mathrm{n}=6$ rats per group); $\mathrm{P}<0.05$ and ${ }^{\# / *} \mathrm{P}<0.01$ between comparison groups, ( $\mathrm{n}=6$ rats per group). CB2R, cannabinoid receptor type 2 .

JWH133 low-dose and high-dose groups, respectively. The expression of autophagy-related proteins in the hippocampus after SE could be tested in all specimens of each group by western blotting. The levels of p-mTOR/mTOR, p-ULK1/ ULK1, Beclin-1, LC3II/LC3I and p62 expression were compared among all groups 1 day after SE (Fig. 4). Compared with the vehicle group, western blotting showed significant and dose-dependent decreases in p-ULK1/ULK1 and p62 expression in the JWH133 low-dose and high-dose groups at 1 day after $\mathrm{SE}(\mathrm{P}<0.05$; Fig. 4$)$ and dose-dependent increases in p-mTOR/mTOR, Beclin-1, and LC3II/LC3I expression in the JWH133 low-dose and high-dose groups 1 day after SE ( $\mathrm{P}<0.05$; Fig. 4). In the JWH133+AM630 group, the results clearly indicated no significant difference in the expression of p-mTOR/mTOR, p-ULK1/ULK1, Beclin-1, LC3II/LC3I and p62 compared with the vehicle group ( $\mathrm{P}>0.05$; Fig. 4). Compared with the JWH133 high-dose group, western blotting showed significant increases in p-ULK1/ULK1 and p62 expression in the JWH133+AM630 group 1 day after SE
( $\mathrm{P}<0.05$; Fig. 4) and decreases in $\mathrm{p}-\mathrm{mTOR} / \mathrm{mTOR}$, Beclin-1, and LC3II/LC3I expression in the JWH133+AM630 group 1 day after $\mathrm{SE}(\mathrm{P}<0.05$; Fig. 4).

Effects of CB2R on the expression of apoptosis-related proteins in the hippocampus after SE. Paralleling the changes in neuronal cell fate determined using a TUNEL assay, the apoptotic process was also altered in the post-SE rat hippocampus. Briefly, the expression of the apoptosis-related protein cleaved caspase- 3 could be detected in all specimens of each group by western blotting. The levels of cleaved caspase-3 were examined in all groups at 1 day after SE. There was no significant difference in the expression of cleaved caspase-3 between the SE and vehicle groups ( $\mathrm{P}>0.05$; Fig. 4). However, the upregulation of cleaved caspase-3 protein was significantly and dose-dependently inhibited in the JWH133-treated group; the reduction in its expression was more marked in the JWH133 high-dose group at 1 day after $\mathrm{SE}(\mathrm{P}<0.05$; Fig. 4). In the JWH133+AM630 group, the results clearly indicated no 

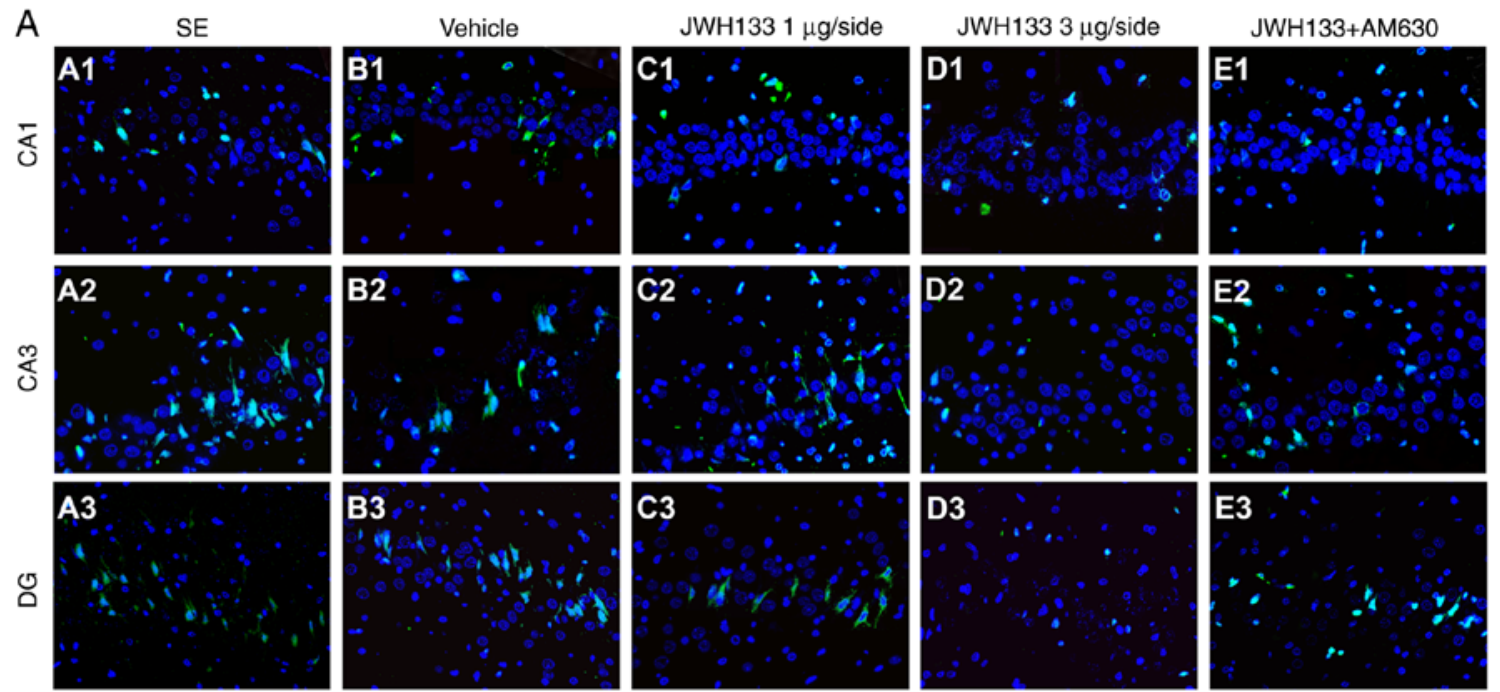

B CA1 region

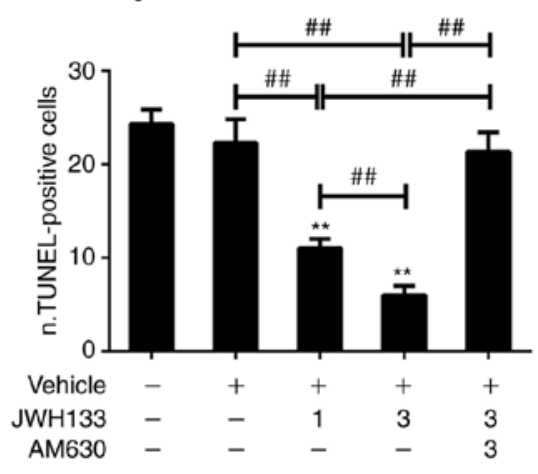

C CA3 region
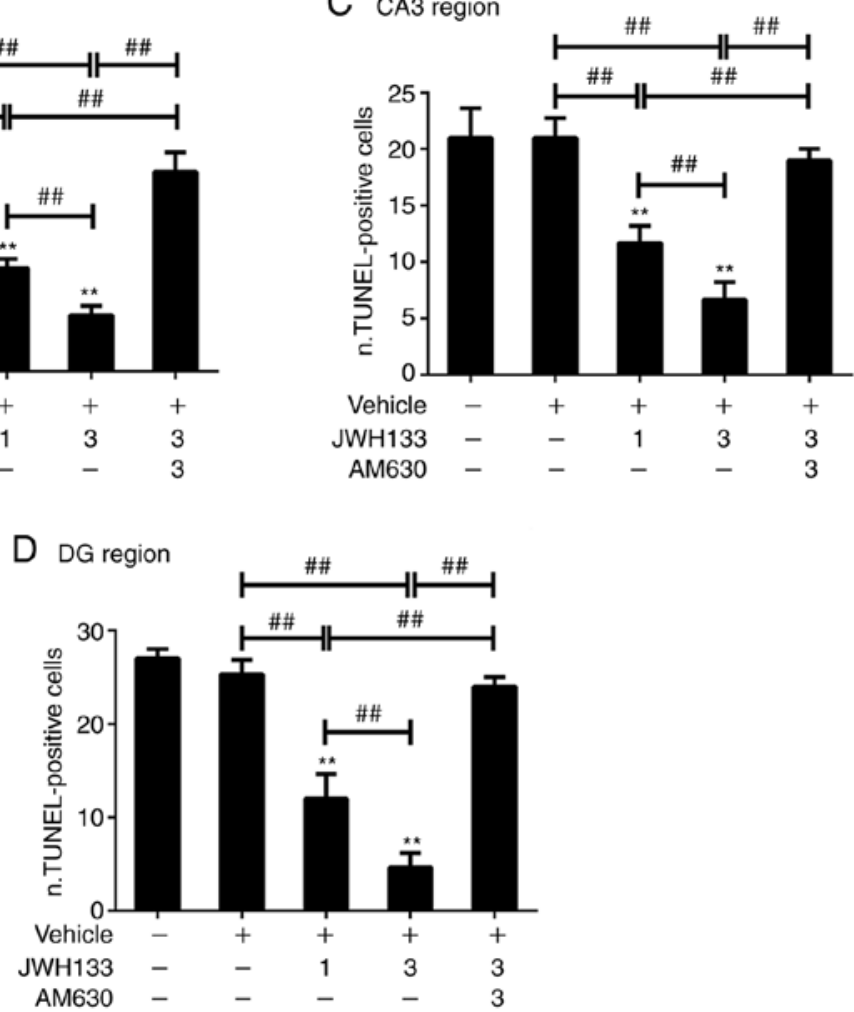

Figure 3. TUNEL staining in the rat hippocampus. (A) TUNEL-positive cells in the different treatment groups and regions are shown. The numbers of TUNEL-positive cells calculated in the (B) CA1, (C) CA3 and (D) DG regions after standard error, respectively. Data are presented as the mean \pm standard deviation. ${ }^{* *} \mathrm{P}<0.01$ vs. the control group, $\left(\mathrm{n}=6\right.$ rats per group); ${ }^{\# \prime} \mathrm{P}<0.01$ vs. comparison groups, $(\mathrm{n}=6$ rats per group). TUNEL, TdT-mediated dUTP nick end labeling.

significant difference in the expression of cleaved caspase-3 compared with the vehicle group ( $\mathrm{P}>0.05$; Fig. 4). Compared with the JWH133 high-dose group, western blotting showed significant increases in cleaved caspase- 3 expression in the JWH133+AM630 group at 1 day after SE (P<0.05; Fig. 4).

\section{Discussion}

Epilepsy as the most common neurological disorder is characterized by the clinical manifestation of 'abnormal excessive or synchronous neuronal activity in the brain' (1). Although the pathogenesis of epilepsy remains unclear, it is generally acknowledged that persistent seizures can directly kill neurons. In addition, a number of studies in previous years has hinted that autophagy and apoptosis contribute to the neuronal damage process through the modulation of Beclin family members and their cascades $(41,42)$. Consistently, the neuronal damage induced by kainate can be worsened by autophagy blockers while being prevented by autophagy inducers $(43,44)$. In detail, mTOR is activated both acutely and chronically after Kainic acid-induced SE, which is prominent in the hippocampus and other cortical areas (45). An early observation from 7 years ago showed an increase in the autophagy markers LC3-II, p-mTOR/mTOR 

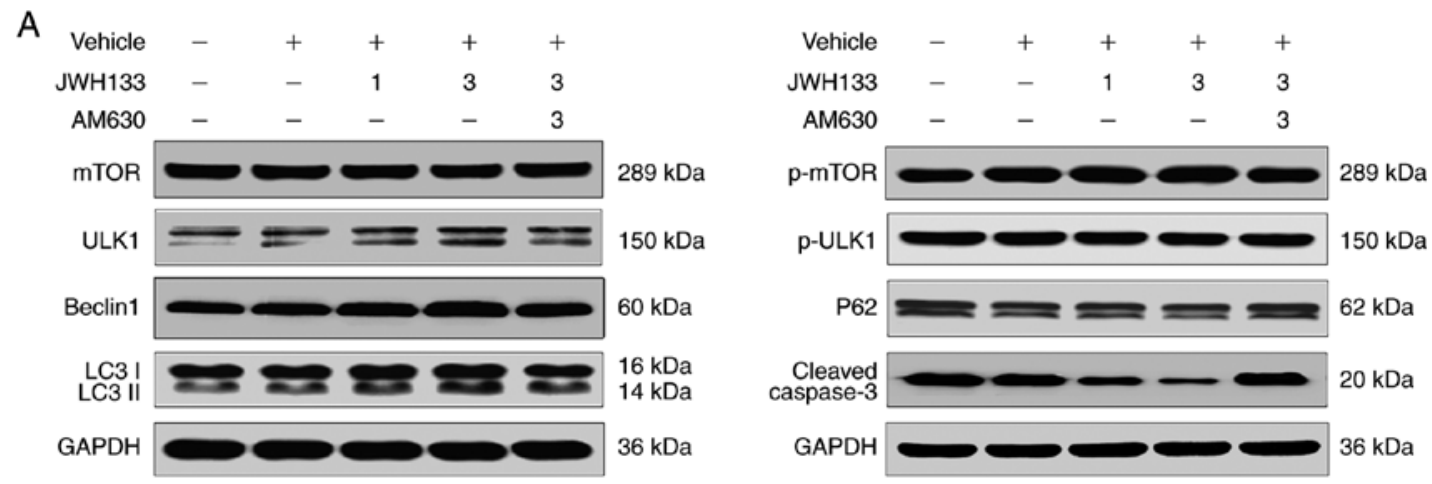

B
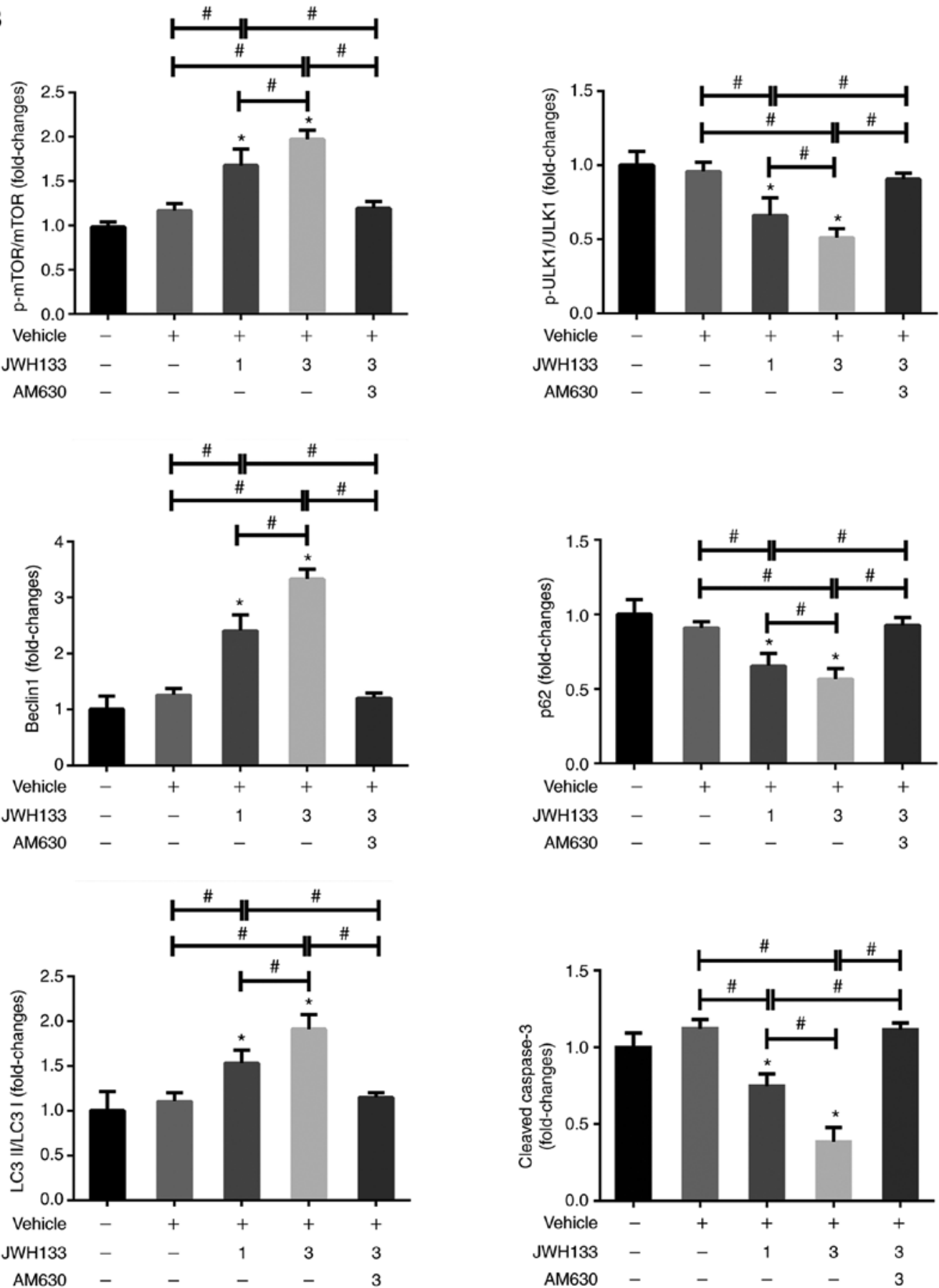

Figure 4. Western blotting was used to simultaneously determine the expression of p-mTOR/mTOR, p-ULK1/ULK1, Beclin-1, p62, LC3II/LC3I and caspase-3 at 1 day post-SE. (A) Immunoblotting results of mTOR, p-mTOR, ULK1, p-ULK1, Beclin-1, p62, LC3II/LC3I and caspase-3 expression at 1 day post-SE. (B) Western blot analysis of p-mTOR/mTOR, p-ULK1/ULK1, Beclin-1, p62, LC3II/LC3I and caspase-3 expression at 1 day post-SE. Data are presented as the mean \pm standard deviation. ${ }^{*} \mathrm{P}<0.05$ vs. the $\mathrm{SE}$ group, $\left(\mathrm{n}=6\right.$ rats per group), ${ }^{\#} \mathrm{P}<0.05$ between comparison groups, ( $\mathrm{n}=6$ rats per group). $\mathrm{SE}$, status epilepticus; p-mTOR, phosphorylated-mammalian target of rapamycin. 
ratio and p-protein kinase $\mathrm{B}(\mathrm{Akt}) / \mathrm{Akt}$ ratio after kainate administration to mice (46). A total of 2 years later, a sudden increase in autophagy markers (LC3II/LC3I and Beclin-1) was also found after pilocarpine administration to rats (47). In this study, it was found that the tendency for a decrease in mTOR expression and an increase in CB2R and LC3 expression was clearest in all three regions at 1 day (the early stage of neuronal repair and damage) after SE. Interestingly, it was hinted that $\mathrm{CB} 2 \mathrm{R}$ may participate in the autophagy process and the expression level of CB2R may be related to the extent of the damage in hippocampal neurons.

Several studies revealed that CB2R exerts neuroprotective effects on alcohol addiction (48), depression (49), cerebral ischemia (50) and Alzheimer's disease (51). The endocannabinoid system also participates in the epileptiform activity in the hippocampus (52-54). Cannabinoid and nitric oxide signaling can interplay in the modulation of hippocampal hyperexcitability in the temporal lobe epilepsy model of rats (55). Carletti et al (56) also found that neuronal nitric oxide synthase is involved in CB/TRPV1 signaling for controlling hippocampal hyperexcitability. Furthermore, a mixed CB1/2 agonist WIN 55,212-2 exerts anti-convulsant effects in acute hypoxia-induced seizures or tonic-clonic seizures evoked by pentylenetetrazole, while a CB2R antagonist AM630 increases pentylenetetrazol-induced seizure severity in a rat model (57). However, Rizzo et al (58) argued that CB2R antagonist AM630 seems to cooperate with WIN 55,212-2 and lowered neuronal hyperexcitability by targeting nitrergic-dependent cGMP pathway. Additionally, Tchekalarova et al (59) discovered that CB2R stimulation by $\beta$-caryophyllene could restrain the spread of seizures. Single administration of JWH133 before $30 \mathrm{~min}$ of pilocarpine treatment postpones the initiation of SE and significantly reduces the frequency of various epileptic behaviors according to the Racine scale (40). The decrease in epilepsy-related scores is in line with the dose-dependent increase in the levels of autophagy-related proteins in the hippocampus of epileptic rats after single administration of JWH133. Because elevation of autophagy-related proteins is related to neural plasticity (60), and epileptic behavior also is related to neural plasticity, autophagy by this mechanism may affect epilepsy-related behaviors. Thus, CB2R may take part in the early stage of pilocarpine-induced SE.

The present results have shown an alteration in the expression of $\mathrm{p}-\mathrm{mTOR} / \mathrm{mTOR}, \mathrm{p}-\mathrm{ULK} 1 / \mathrm{ULK} 1$, Beclin-1, LC3II/LC3I and p62 proteins for autophagy and caspase-3 proteins for apoptosis in CA1, CA3, and DG regions of the rat hippocampus after SE. In short, the expression levels of the autophagy-related proteins p-mTOR/mTOR, Beclin-1 and LC3II/LC3I were dose-dependently increased in the rat hippocampus 1 day after SE especially in the JWH133 high-dose group. At the same time, the expression levels of the autophagy proteins p-ULK1/ULK1 and p62 were downregulated. The results were reversed by pretreament with the CB2R antagonist, AM630. A dose-dependent decrease in the number of apoptotic neurons and the expression of the apoptosis protein cleaved caspase-3 was evident at 1 day after SE in the JWH133 treated group, and was further decreased in the group receiving the highest dose of JWH133. In the present study, intracerebroventricular injections (JWH133, 1 or $3 \mu \mathrm{g}$ in $1 \mu \mathrm{l} /$ side; AM630, $3 \mu \mathrm{g}$ in $1 \mu \mathrm{l} /$ side) was used and an AM630 alone group was not used. There are two reasons why the groups that were used were chosen. One reason is that some conclusions are obtained according to the references. Bilateral microinjections of JWH133 (1 or $3 \mu \mathrm{g}$ in $1 \mu \mathrm{l} /$ side) into the ventral tegmental area significantly reduced cocaine self-administration in WT mice and this effect was blocked by coadministration of AM630 and JWH133, whereas microinjections of AM630 alone had no effect on cocaine self-administration (61). Administration of AM630 alone exerts no effects on hippocampal hyperexcitability in the maximal dentate activation model of hippocampal epilepsy (58). The other reason is that this paper mainly focuses on the effect of CB2R agonist JWH133 on neuronal autophagy in the early stage of neuronal damage and repair after SE, to find a new target for epilepsy treatment. CB2R antagonist AM630 is only used to antagonize the effect of the agonist JWH133. In summary, the results from the present study clearly demonstrated that CB2R significantly reduced neuronal damage in the rat hippocampus after SE through the induction of autophagy and inhibition of apoptosis. Furthermore, the results from this study also suggest that the CB2R could be promising for use in the treatment of epilepsy.

Autophagy can exert both harmful and beneficial effects in different situations (62-65). The present results suggests that the increase in autophagy signaling induced by SE or seizure attacks has a protective role in hippocampal neurons, while apoptosis signaling seems to lead to neuronal death in the hippocampus after SE. Furthermore, the current study hints that apoptosis can be considered an outcome following autophagy failure, because these two mechanisms cooperate to influence hippocampal neuronal fate after SE. In other words, autophagy acts as a self-protection mechanism to keep neuronal dynamic equilibrium at early stages (about $24 \mathrm{~h}$ ) post-SE, however, apoptosis is activated to ameliorate neuron death when hippocampal neuronal autophagy is insufficient. The autophagy and apoptosis mechanism participating in hippocampal neuronal damage after SE is still unclear. The present results only reveal that $\mathrm{CB} 2 \mathrm{R}$ may be involved in neuronal damage and CB2R stimulation may protect hippocampal neurons by modulating autophagy and apoptosis.

Of course, the paper has a limit in terms of scope. The mTOR plays multiple roles in neuronal and non-neuronal cells development, neuronal plasticity, as well as in the expression of different neuronal molecules involved in cell excitability. These effects may not be directly dependent on autophagy (66). Thus, attribution of mTOR-related epilepsy to autophagy impairment could remain a mere speculation, if not supported by the analyses on the other molecular target implicated in autophagy such as LC3 and ULK1 as was inserted in the present study (67). Regarding this point, the 'ATG7 KO' strain of mice which possess a normal mTOR signaling but disrupted autophagy activity is being considered for use in the present study (68) and the relevant experiments have already been started.

In summary, the present study indicated that CB2R orchestrates neuronal autophagy through regulation of the mTOR signaling pathway in the hippocampus of developing rats with SE. These novel findings might provide an important basis for further investigation of the therapeutic role of CB2R in the treatment or prevention of epilepsy-related neuronal damage. 


\section{Acknowledgements}

Not applicable.

\section{Funding}

The present study was supported by grants from the Department of Science and Technology of Liaoning Province, China (grant nos. 2014225007 and 2019-BS-280), the Department of Education and Technology of Liaoning Province, China (grant no. L2014309) and the National Key Research and Development Program of China (grant no. 2016YFC1306203).

\section{Availability of data and materials}

The datasets used and/or analyzed during the current study are available from the corresponding author on reasonable request.

\section{Authors' contributions}

QW, MZ and HW were involved in the conception and design of the study and the drafting of the manuscript. QW and MZ were involved in the analysis and interpretation of the data. JZ and XL assisted in setting up the protocols as well as in their administration, and XL offered financial support for the project leading to this publication. QW and MZ performed all the experiments and acquired all data. All authors read and approved the manuscript.

\section{Ethics approval and consent to participate}

The China Medical University Institutional Animal Care and Use Committee (no. 2016PS260K) approved all procedures.

\section{Patient consent for publication}

Not applicable.

\section{Competing interests}

The authors declare that they have no competing interests.

\section{References}

1. Fisher RS, Acevedo C, Arzimanoglou A, Bogacz A, Cross JH, Elger CE, Engel J Jr, Forsgren L, French JA, Glynn M, et al: ILAE official report: A practical clinical definition of epilepsy. Epilepsia 55: 475-482, 2014.

2. Engel J Jr: Mesial temporal lobe epilepsy: What have we learned? Neuroscientist 7: 340-352, 2001.

3. Shibley H and Smith BN: Pilocarpine-induced status epilepticus results in mossy fiber sprouting and spontaneous seizures in C57BL/6 and CD-1 mice. Epilepsy Res 49: 109-120, 2002.

4. Morimoto K, Fahnestock M and Racine RJ: Kindling and status epilepticus models of epilepsy: Rewiring the brain. Prog Neurobiol 73: 1-60, 2004.

5. Friedman WJ: Proneurotrophins, seizures, and neuronal apoptosis. Neuroscientist 16: 244-252, 2010.

6. Baram TZ, Jensen FE and Brooks-Kayal A: Does acquired epileptogenesis in the immature brain require neuronal death. Epilepsy Curr 11: 21-26, 2011.

7. Varvel NH, Neher JJ, Bosch A, Wang W, Ransohoff RM, Miller RJ and Dingledine R: Infiltrating monocytes promote brain inflammation and exacerbate neuronal damage after status epilepticus. Proc Natl Acad Sci USA 113: E5665-E5674, 2016.
8. Hung SY, Huang WP, Liou HC and Fu WM: LC3 overexpression reduces $A \beta$ neurotoxicity through increasing $\alpha 7 \mathrm{nAchR}$ expression and autophagic activity in neurons and mice. Neuropharmacology 93: 243-251, 2015

9. Vieira M, Fernandes J, Carreto L, Anuncibay-Soto B, Santos M, Han J, Fernández-López A, Duarte CB, Carvalho AL and Santos AE: Ischemic insults induce necroptotic cell death in hippocampal neurons through the up-regulation of endogenous RIP3. Neurobiol Dis 68: 26-36, 2014.

10. $\mathrm{Xu} \mathrm{M}$ and Zhang HL: Death and survival of neuronal and astrocytic cells in ischemic brain injury: A role of autophagy. Acta Pharmacol Sin 32: 1089-1099, 2011.

11. Hochfeld M, Lamecker H, Thomale UW, Schulz M, Zachow S and Haberl H: Frame-based cranial reconstruction. J Neurosurg Pediatr 13: 319-323, 2014.

12. Ravikumar B, Vacher C, Berger Z, Davies JE, Luo S, Oroz LG, Scaravilli F, Easton DF, Duden R, O'Kane CJ and Rubinsztein DC: Inhibition of mTOR induces autophagy and reduces toxicity of polyglutamine expansions in fly and mouse models of Huntington disease. Nat Genet 36: 585-595, 2004.

13. Hoeffer CA and Klann E: mTOR signaling: At the crossroads of plasticity, memory and disease. Trends Neurosci 33: 67-75, 2010.

14. Costa-Mattioli M, Sossin WS, Klann E and Sonenberg N: Translational control of long-lasting synaptic plasticity and memory. Neuron 61: 10-26, 2009.

15. Laplante M and Sabatini DM: mTOR signaling in growth control and disease. Cell 149: 274-293, 2012.

16. Jung CH, Jun CB, Ro SH, Kim YM, Otto NM, Cao J, Kundu M and Kim DH: ULK-Atg13-FIP200 complexes mediate mTOR signaling to the autophagy machinery. Mol Biol Cell 20: 1992-2003, 2009.

17. Russell RC, Tian Y, Yuan H, Park HW, Chang YY, Kim J, Kim H, Neufeld TP, Dillin A and Guan KL: ULK1 induces autophagy by phosphorylating Beclin-1 and activating VPS34 lipid kinase. Nat Cell Biol 15: 741-750, 2013.

18. Rubinsztein DC, Codogno P and Levine B: Autophagy modulation as a potential therapeutic target for diverse diseases. Nat Rev Drug Discov 11: 709-730, 2012.

19. Harris H and Rubinsztein DC: Control of autophagy as a therapy for neurodegenerative disease. Nat Rev Neurol 8: 108-117, 2011.

20. Pankiv S, Clausen TH, Lamark T, Brech A, Bruun JA, Outzen H, Øvervatn A, Bjørkøy G and Johansen T: p62/SQSTM1 binds directly to Atg8/LC3 to facilitate degradation of ubiquitinated protein aggregates by autophagy. J Biol Chem 282: 24131-24145, 2007.

21. Hara T, Nakamura K, Matsui M, Yamamoto A, Nakahara Y, Suzuki-Migishima R, Yokoyama M, Mishima K, Saito I, Okano H and Mizushima N: Suppression of basal autophagy in neural cells causes neurodegenerative disease in mice. Nature 441: 885-889, 2006.

22. Menzies FM, Fleming A, Caricasole A, Bento CF, Andrews SP, Ashkenazi A, Füllgrabe J, Jackson A, Jimenez Sanchez M, Karabiyik C, et al: Autophagy and neurodegeneration: Pathogenic mechanisms and therapeutic opportunities. Neuron 93: 1015-1034, 2017.

23. Pasquali L, Longone P, Isidoro C, Ruggieri S, Paparelli A and Fornai F: Autophagy, lithium, and amyotrophic lateral sclerosis. Muscle Nerve 40: 173-194, 2009.

24. Fornai F, Longone P, Ferrucci M, Lenzi P, Isidoro C, Ruggieri S and Paparelli A: Autophagy and amyotrophic lateral sclerosis: The multiple roles of lithium. Autophagy 4: 527-530, 2008.

25. Hwang JY, Gertner M, Pontarelli F, Court-Vazquez B, Bennett MV, Ofengeim D and Zukin RS: Global ischemia induces lysosomal-mediated degradation of mTOR and activation of autophagy in hippocampal neurons destined to die. Cell Death Differ 24: 317-329, 2017.

26. Hosseinzadeh M, Nikseresht S, Khodagholi F, Naderi N and Maghsoudi N: Cannabidiol post-treatment alleviates rat epileptic-related behaviors and activates hippocampal cell autophagy pathway along with antioxidant defense in chronic phase of pilocarpine-induced seizure. J Mol Neurosci 58: 432-440, 2016.

27. Soltesz I, Alger BE, Kano M, Lee SH, Lovinger DM, Ohno-Shosaku T and Watanabe M: Weeding out bad waves: Towards selective cannabinoid circuit control in epilepsy. Nat Rev Neurosci 16: 264-277, 2015.

28. Regehr WG, Carey MR and Best AR: Activity-dependent regulation of synapses by retrograde messengers. Neuron 63: 154-170, 2009.

29. Jansen EM, Haycock DA, Ward SJ and Seybold VS: Distribution of cannabinoid receptors in rat brain determined with aminoalkylindoles. Brain Res 575: 93-102, 1992. 
30. Muñoz-Luque J, Ros J, Fernández-Varo G, Tugues S Morales-Ruiz M, Alvarez CE, Friedman SL, Arroyo V and Jiménez W: Regression of fibrosis after chronic stimulation of cannabinoid CB2 receptor in cirrhotic rats. J Pharmacol Exp Ther 324: 475-483, 2008.

31. Galiègue S, Mary S, Marchand J, Dussossoy D, Carrière D, Carayon P, Bouaboula M, Shire D, Le Fur G and Casellas P: Expression of central and peripheral cannabinoid receptors in human immune tissues and leukocyte subpopulations. Eur J Biochem 232: 54-61, 1995.

32. Li Y and Kim J: Neuronal expression of CB2 cannabinoid receptor mRNAs in the mouse hippocampus. Neuroscience 311: 253-267, 2015

33. Lanciego JL, Barroso-Chinea P, Rico AJ, Conte-Perales L, Callén L, Roda E, Gómez-Bautista V, López IP, Lluis C, Labandeira-García JL and Franco R: Expression of the mRNA coding the cannabinoid receptor 2 in the pallidal complex of Macaca fascicularis. J Psychopharmacol 25: 97-104, 2011.

34. Viscomi MT, Oddi S, Latini L, Pasquariello N, Florenzano F, Bernardi G, Molinari M and Maccarrone M: Selective CB2 receptor agonism protects central neurons from remote axotomy-induced apoptosis through the PI3K/Akt pathway. J Neurosci 29: 4564-4570, 2009

35. Kim J and Li Y: Chronic activation of CB2 cannabinoid receptors in the hippocampus increases excitatory synaptic transmission. J Physiol 593: 871-886, 2015.

36. Stempel AV, Stumpf A, Zhang HY, Özdoğan T, Pannasch U, Theis AK, Otte DM, Wojtalla A, Rácz I, Ponomarenko A, et al: Cannabinoid type 2 receptors mediate a cell type-specific plasticity in the hippocampus. Neuron 90: 795-809, 2016.

37. Sugaya Y, Yamazaki M, Uchigashima M, Kobayashi K, Watanabe M, Sakimura K and Kano M: Crucial roles of the endocannabinoid 2-arachidonoylglycerol in the suppression of epileptic seizures. Cell Rep 16: 1405-1415, 2016.

38. Wu Q and Wang $\mathrm{H}$ : The spatiotemporal expression changes of $\mathrm{CB} 2 \mathrm{R}$ in the hippocampus of rats following pilocarpine-induced status epilepticus. Epilepsy Res 148: 8-16, 2018.

39. Curia G, Longo D, Biagini G, Jones RS and Avoli M: The pilocarpine model of temporal lobe epilepsy. J Neurosci Methods 172: 143-157, 2008

40. Racine RJ: Modification of seizure activity by electrical stimulation. II. Motor seizure. Electroencephalogr Clin Neurophysiol 32: 281-294, 1972.

41. Li X, Lou X, Xu S, Wang Q, Shen M and Miao J: Knockdown of miR-372 inhibits nerve cell apoptosis induced by spinal cord ischemia/reperfusion injury via enhancing autophagy by up-regulating Beclin-1. J Mol Neurosci 66: 437-444, 2018.

42. Salminen A, Kaarniranta K, Kauppinen A, Ojala J, Haapasalo A, Soininen $\mathrm{H}$ and Hiltunen M: Impaired autophagy and APP processing in Alzheimer's disease: The potential role of Beclin 1 interactome. Prog Neurobiol 106-107: 33-54, 2013.

43. Fornai F, Longone P, Cafaro L, Kastsiuchenka O, Ferrucci M, Manca ML, Lazzeri G, Spalloni A, Bellio N, Lenzi P, et al: Lithium delays progression of amyotrophic lateral sclerosis. Proc Natl Acad Sci USA 105: 2052-2057, 2008.

44. Calderó J, Brunet N, Tarabal O, Piedrafita L, Hereu M, Ayala V and Esquerda JE: Lithium prevents excitotoxic cell death of motoneurons in organotypic slice cultures of spinal cord. Neuroscience 165: 1353-1369, 2010.

45. Macias M, Blazejczyk M, Kazmierska P, Caban B, Skalecka A, Tarkowski B, Rodo A, Konopacki J and Jaworski J: Spatiotemporal characterization of mTOR kinase activity following kainic acid induced status epilepticus and analysis of rat brain response to chronic rapamycin treatment. PLoS One 8: e64455, 2013.

46. Shacka JJ, Lu J, Xie ZL, Uchiyama Y, Roth KA and Zhang J: Kainic acid induces early and transient autophagic stress in mouse hippocampus. Neurosci Lett 414: 57-60, 2007.

47. Cao L, Xu J, Lin Y, Zhao X, Liu X and Chi Z: Autophagy is upregulated in rats with status epilepticus and partly inhibited by Vitamin E. Biochem Biophys Res Commun 379: 949-953, 2009.

48. Al Mansouri S, Ojha S, Al Maamari E, Al Ameri M, Nurulain SM and Bahi A: The cannabinoid receptor 2 agonist, $\beta$-caryophyllene, reduced voluntary alcohol intake and attenuated ethanol-induced place preference and sensitivity in mice. Pharmacol Biochem Behav 124: 260-268, 2014.

49. Katsuyama S, Mizoguchi H, Kuwahata H, Komatsu T, Nagaoka K, Nakamura H, Bagetta G, Sakurada T and Sakurada S: Involvement of peripheral cannabinoid and opioid receptors in $\beta$-caryophyllene-induced antinociception. Eur J Pain 17: 664-675, 2013.
50. Choi IY, Ju C, Anthony Jalin AM, Lee DI, Prather PL and Kim WK: Activation of cannabinoid CB2 receptor-mediated AMPK/CREB pathway reduces cerebral ischemic injury. Am J Pathol 182: 928-939, 2013.

51. Cheng Y, Dong Z and Liu S: $\beta$-Caryophyllene ameliorates the Alzheimer-like phenotype in APP/PS1 Mice through CB2 receptor activation and the PPAR $\gamma$ pathway. Pharmacology 94: 1-12, 2014.

52. Wallace MJ, Martin BR and DeLorenzo RJ: Evidence for a physiological role of endocannabinoids in the modulation of seizure threshold and severity. Eur J Pharmacol 452: 295-301, 2002.

53. Monory K, Massa F, Egertová M, Eder M, Blaudzun H, Westenbroek R, Kelsch W, Jacob W, Marsch R, Ekker M, et al: The endocannabinoid system controls key epileptogenic circuits in the hippocampus. Neuron 51: 455-466, 2006.

54. Ludányi A, Eross L, Czirják S, Vajda J, Halász P, Watanabe M, Palkovits M, Maglóczky Z, Freund TF and Katona I: Downregulation of the $\mathrm{CB} 1$ cannabinoid receptor and related molecular elements of the endocannabinoid system in epileptic human hippocampus. J Neurosci 28: 2976-2990, 2008.

55. Carletti F, Gambino G, Rizzo V, Ferraro G and Sardo P: Cannabinoid and nitric oxide signaling interplay in the modulation of hippocampal hyperexcitability: Study on electrophysiological and behavioral models of temporal lobe epilepsy in the rat. Neuroscience 303: 149-159, 2015.

56. Carletti F, Gambino G, Rizzo V, Ferraro G and Sardo P: Neuronal nitric oxide synthase is involved in CB/TRPV1 signalling: Focus on control of hippocampal hyperexcitability. Epilepsy Res 138: 18-25, 2017.

57. Huizenga MN, Wicker E, Beck VC and Forcelli PA: Anticonvulsant effect of cannabinoid receptor agonists in models of seizures in developing rats. Epilepsia 58: 1593-1602, 2017.

58. Rizzo V, Carletti F, Gambino G, Schiera G, Cannizzaro C, Ferraro G and Sardo P: Role of CB2 receptors and cGMP pathway on the cannabinoid-dependent antiepileptic effects in an in vivo model of partial epilepsy. Epilepsy Res 108: 1711-1718, 2014.

59. Tchekalarova J, da Conceição Machado K, Gomes Júnior AL, de Carvalho Melo Cavalcante AA, Momchilova A and Tzoneva R: Pharmacological characterization of the cannabinoid receptor 2 agonist, $\beta$-caryophyllene on seizure models in mice. Seizure 57: 22-26, 2018

60. Otabe H, Nibuya M, Shimazaki K, Toda H, Suzuki G, Nomura S and Shimizu K: Electroconvulsive seizures enhance autophagy signaling in rat hippocampus. Prog Neuropsychopharmacol Biol Psychiatry 50: 37-43, 2014.

61. Zhang HY, Gao M, Liu QR, Bi GH, Li X, Yang HJ, Gardner EL, $\mathrm{Wu} \mathrm{J}$ and $\mathrm{Xi} \mathrm{ZX}$ : Cannabinoid CB2 receptors modulate midbrain dopamine neuronal activity and dopamine-related behavior in mice. Proc Natl Acad Sci USA 111: E5007-E5015, 2014.

62. Gump JM and Thorburn A: Autophagy and apoptosis: What's the connection? Trends Cell Biol 21: 387-392, 2011.

63. Leber B and Andrews DW: Closing in on the link between apoptosis and autophagy. F1000 Biol Rep 2: 88, 2010.

64. Linkermann A and Green DR: Necroptosis. N Engl J Med 370: 455-465, 2014.

65. Nikoletopoulou V, Markaki M, Palikaras K and Tavernarakis N: Crosstalk between apoptosis, necrosis and autophagy. Biochim Biophys Acta 1833: 3448-3459, 2013.

66. Lasarge CL and Danzer SC: Mechanisms regulating neuronal excitability and seizure development following mTOR pathway hyperactivation. Front Mol Neurosci 7: 18, 2014.

67. Giorgi FS, Biagioni F, Lenzi P, Frati A and Fornai F: The role of autophagy in epileptogenesis and in epilepsy-induced neuronal alterations. J Neural Transm (Vienna) 122: 849-862, 2015.

68. McMahon J, Huang X, Yang J, Komatsu M, Yue Z, Qian J, Zhu X and Huang Y: Impaired autophagy in neurons after disinhibition of mammalian target of rapamycin and its contribution to epileptogenesis. J Neurosci 32: 15704-15714, 2012.

This work is licensed under a Creative Commons Attribution-NonCommercial-NoDerivatives 4.0 International (CC BY-NC-ND 4.0) License. 